\title{
Research Article \\ Effects of Dispersal for a Logistic Growth Population in Random Environments
}

\author{
Xiaoling Zou, ${ }^{1}$ Dejun Fan, ${ }^{1}$ and Ke Wang ${ }^{1,2}$ \\ ${ }^{1}$ Department of Mathematics, Harbin Institute of Technology (Weihai), Weihai 264209, China \\ ${ }^{2}$ School of Mathematics and Statistics, Northeast Normal University, Changchun 130024, China \\ Correspondence should be addressed to Dejun Fan; fandejun2009@yahoo.com.cn
}

Received 23 November 2012; Accepted 12 February 2013

Academic Editor: Julio Rossi

Copyright (C) 2013 Xiaoling Zou et al. This is an open access article distributed under the Creative Commons Attribution License, which permits unrestricted use, distribution, and reproduction in any medium, provided the original work is properly cited.

\begin{abstract}
We study a stochastic logistic model with diffusion between two patches in this paper. Using the definition of stationary distribution, we discuss the effect of dispersal in detail. If the species are able to have nontrivial stationary distributions when the patches are isolated, then they continue to do so for small diffusion rates. In addition, we use some examples and numerical experiments to reflect that diffusions are capable of both stabilizing and destabilizing a given ecosystem.
\end{abstract}

\section{Introduction}

Dispersal is a ubiquitous phenomenon in the natural world. This phenomenon plays a very important role in understanding the ecological and evolutionary dynamics of populations. The theoretical studies of spatial distributions can be traced back as far as Skellam [1]. Then many scholars have focused on the effects of spatial factors which play a crucial role in the study of stability. Some mathematical models dealt with a single population dispersing among patches (see [2-9] and references cited therein). The others dealt with competition or predator-prey interactions in patchy environments (see [10-16] and references cited therein). These models centered round local and global stability of equilibrium points, persistence, and extinction of populations.

Through the studies for the diffusion systems and the corresponding ones without diffusion, many authors have discussed the relationship between the existence of the equilibriums and their stability. Levin [10] showed that two unstable competitive patches can be stabilized by diffusion; Levin [11] also showed that diffusion can destabilize a stable system by using a prey-predator model; Allen [4] proved that a single species diffusion system remains weakly persistent if the strength of diffusion is small enough; Beretta and Takeuchi $[5,6]$ showed that small diffusion cannot change the global stability of the model. Takeuchi also proved that diffusion among patches will not destabilize singlepopulation dynamics [9].

However, the most natural phenomena do not follow strictly deterministic laws, but rather oscillate randomly about some averages. That is to say populations in the real word are inevitably affected by various environmental noises which is an important phenomenon in ecosystems [17-19]. So we will consider a stochastic diffusion system which is composed of two patches and connected by diffusion. Then we want to know "how are the effects of dispersal under random environments?" According to the author's best knowledge, there are few results dealing with this problem, and stabilizing and/or destabilizing effects of dispersal remain largely unknown due to difficulties involved by random disturbances. Generally speaking, there does not have time independent equilibrium point for a stochastic system. Hence we will investigate the effects of dispersal by the concept of stationary distribution (some analogue which plays the role of the deterministic equilibrium point and reflects the stability to some extent). In this paper, we will show that diffusion cannot change the existence of stable stationary distribution for the stochastic model if the strength of diffusion is small enough. Moreover, small diffusion rates have some stabilizing effects, and large diffusion rates have some destabilizing effects on the stochastic model. That is, diffusions are capable of both stabilizing and destabilizing a given ecosystem. 


\section{Formulation of the Mathematical Model}

The classical mathematical model describing the dynamics of a single species is the logistic model, governed by the following differential equation:

$$
\mathrm{d} x(t)=x(t)(r-k x(t)) \mathrm{d} t .
$$

This is a very popular model, and many scholars have considered various ecosystems based on this equation. If we take the dispersal phenomenon into consideration, a single population dispersing in two patches becomes

$$
\begin{aligned}
& \mathrm{d} x_{1}=\left[x_{1}\left(r_{1}-k_{1} x_{1}\right)+\varepsilon_{12}\left(x_{2}-x_{1}\right)\right] \mathrm{d} t, \\
& \mathrm{~d} x_{2}=\left[x_{2}\left(r_{2}-k_{2} x_{2}\right)+\varepsilon_{21}\left(x_{1}-x_{2}\right)\right] \mathrm{d} t,
\end{aligned}
$$

where $x_{i}$ represents the population density of the species in $i$ th patch. $r_{i}$ and $k_{i}$ are the growth rate and self-competition coefficient of the population in the $i$ th patch. $\varepsilon_{i j}$ is a nonnegative diffusion coefficient for the species from $j$ th patch to $i$ th patch $(i \neq j)$. It is supposed that the net exchange from $i$ th patch to $j$ th patch is proportional to the difference of population densities $x_{i}-x_{j}$ in each patch as the usual assumption (see $[2,4-6,20,21]$ ).

Taking the effect of randomly fluctuating environment into consideration, we incorporate white noises in deterministic models. We assume that fluctuations in the environments will manifest themselves mainly as fluctuations in the growth rates of the populations. We usually estimate them by average values plus error terms which follow normal distributions in practice. Let

$$
\begin{aligned}
& r_{1} \longrightarrow r_{1}+\sigma_{1} \dot{B}_{1}(t), \\
& r_{2} \longrightarrow r_{2}+\sigma_{2} \dot{B}_{2}(t),
\end{aligned}
$$

where $B_{1}(t), B_{2}(t)$ are mutually independent Brownian motions and $\sigma_{1}$ and $\sigma_{2}$ reflect the intensities of the white noises. Then, the corresponding Itô-type stochastic system which takes the dispersal phenomenon into consideration becomes

$$
\begin{aligned}
& \mathrm{d} x_{1}=\left[x_{1}\left(r_{1}-k_{1} x_{1}\right)+\varepsilon_{12}\left(x_{2}-x_{1}\right)\right] \mathrm{d} t+\sigma_{1} x_{1} \mathrm{~d} B_{1}(t), \\
& \mathrm{d} x_{2}=\left[x_{2}\left(r_{2}-k_{2} x_{2}\right)+\varepsilon_{21}\left(x_{1}-x_{2}\right)\right] \mathrm{d} t+\sigma_{2} x_{2} \mathrm{~d} B_{2}(t) .
\end{aligned}
$$

Throughout this paper, unless otherwise specified, we let $\left(\Omega, F,\{\mathscr{F}\}_{t \geq 0}, P\right)$ be a complete probability space. $\{\mathscr{F}\}_{t \geq 0}$ is a filtration defined on this space satisfying the usual conditions (It is right continuous, and $\mathscr{F}_{0}$ contains all $P$-null sets.).

\section{Existence and Uniqueness of the Positive Solution for System (4)}

Population densities $x_{1}(t)$ and $x_{2}(t)$ should be nonnegative by their biological significance. For this reason, we want to study system (4) in the region

$$
R_{+}^{2}=\left\{\left(x_{1}, x_{2}\right) \in R^{2} \mid x_{1}>0, x_{2}>0\right\} .
$$

Now, we will show that $R_{+}^{2}$ is a positive invariant set.
Theorem 1. For any initial value $\left(x_{1}(0), x_{2}(0)\right) \in R_{+}^{2}$, there is a unique solution $\left(x_{1}(t), x_{2}(t)\right)$ to system (4) on $t \geq 0$, and the solution will remain in $R_{+}^{2}$ with probability 1 .

Proof. Our proof is motivated by the works of Mao et al. [22]. All the coefficients in system (4) are locally Lipschitz continuous; then for any given initial value $\left(x_{1}(0), x_{2}(0)\right) \in$ $R_{+}^{2}$, there is a unique maximal local solution $\left(x_{1}(t), x_{2}(t)\right)$ on $t \in\left[0, \tau_{e}\right]$, where $\tau_{e}$ is an explosion time (see e.g. [23, 24]). In order to show this solution is global, we only need to prove $\tau_{e}=\infty$. Let $k_{0}>0$ be so large that $x_{i}(0), i=1,2$ lying within the interval $\left[1 / k_{0}, k_{0}\right]$. For each integer $k>k_{0}$, define stopping times as follows:

$$
\tau_{k}=\inf \left\{t \in\left[0, \tau_{e}\right]: x_{i}(t) \notin\left(\frac{1}{k}, k\right) \text { for some } i=1,2\right\} \text {. }
$$

It is easy to see $\tau_{k}$ is increasing as $k \rightarrow \infty$. Set $\tau_{\infty}=$ $\lim _{k \rightarrow \infty} \tau_{k}$; hence $\tau_{\infty} \leq \tau_{e}$ a.s. If we can prove $\tau_{\infty}=\infty$ a.s., then $\tau_{e}=\infty$ a.s. and $\left(x_{1}(t), x_{2}(t)\right) \in R_{+}^{2}$ a.s. for all $t \geq 0$. In other words, we only need to prove $\tau_{\infty}=\infty$ a.s.. For if this statement is false, then there are two constants $T>0$ and $\varepsilon \in(0,1)$ such that

$$
P\left\{\tau_{\infty} \leq T\right\}>\varepsilon
$$

Consequently, there is an integer $k_{1} \geq k_{0}$ satisfying

$$
P\left\{\tau_{k} \leq T\right\} \geq \mathcal{E}
$$

for all $k \geq k_{1}$. Define a $C^{2}$-function $V: R_{+}^{2} \rightarrow R_{+}$by

$$
V\left(x_{1}, x_{2}\right)=\left(\sqrt{x_{1}}-1-0.5 \ln x_{1}\right)+\left(\sqrt{x_{2}}-1-0.5 \ln x_{2}\right) .
$$

The nonnegativity of this function can be seen from

$$
\sqrt{y}-1-0.5 \ln y \geq 0, \quad \text { on } y>0 \text {. }
$$

If $\left(x_{1}, x_{2}\right) \in R_{+}^{2}$, Itô's formula shows that

$\mathrm{d} V$

$$
\begin{aligned}
= & \frac{\partial V}{\partial x_{1}} \mathrm{~d} x_{1}+\frac{\partial V}{\partial x_{2}} \mathrm{~d} x_{2}+\frac{1}{2} \frac{\partial^{2} V}{\partial^{2} x_{1}} \sigma_{1}^{2} x_{1}^{2}+\frac{1}{2} \frac{\partial^{2} V}{\partial^{2} x_{2}} \sigma_{2}^{2} x_{2}^{2} \\
= & 0.5\left(x_{1}^{-0.5}-x_{1}^{-1}\right)\left[x_{1}\left(r_{1}-k_{1} x_{1}\right)+\varepsilon_{12}\left(x_{2}-x_{1}\right)\right] \mathrm{d} t \\
& +0.5\left(x_{2}^{-0.5}-x_{2}^{-1}\right) \\
& \times\left[x_{2}\left(r_{2}-k_{2} x_{2}\right)+\varepsilon_{21}\left(x_{1}-x_{2}\right)\right] \mathrm{d} t \\
& +0.5\left(-0.25 x_{1}^{-1.5}+0.5 x_{1}^{-2}\right) \sigma_{1}^{2} x_{1}^{2} \mathrm{~d} t \\
& +0.5\left(-0.25 x_{2}^{-1.5}+0.5 x_{2}^{-2}\right) \sigma_{2}^{2} x_{2}^{2} \mathrm{~d} t+0.5\left(x_{1}^{-0.5}-x_{1}^{-1}\right) \\
& \times \sigma_{1} x_{1} \mathrm{~d} B_{1}(t)+0.5\left(x_{2}^{-0.5}-x_{2}^{-1}\right) \sigma_{2} x_{2} \mathrm{~d} B_{2}(t)
\end{aligned}
$$




$$
\begin{aligned}
= & -0.5 k_{1} x_{1}^{1.5}+0.5 k_{1} x_{1}+0.5\left(r_{1}-\varepsilon_{12}-0.25 \sigma_{1}^{2}\right) x_{1}^{0.5} \\
& \left.-0.5\left(r_{1}-\varepsilon_{12}-0.5 \sigma_{1}^{2}\right)\right] \mathrm{d} t \\
+ & {\left[-0.5 k_{2} x_{2}^{1.5}+0.5 k_{2} x_{2}+0.5\left(r_{2}-\varepsilon_{21}-0.25 \sigma_{2}^{2}\right) x_{2}^{0.5}\right.} \\
& \left.\quad-0.5\left(r_{2}-\varepsilon_{21}-0.5 \sigma_{2}^{2}\right)\right] \mathrm{d} t \\
& +\left[0.5\left(x_{1}^{-0.5}-x_{1}^{-1}\right) \varepsilon_{12} x_{2}+0.5\left(x_{2}^{-0.5}-x_{2}^{-1}\right) \varepsilon_{21} x_{1}\right] \mathrm{d} t \\
+ & 0.5\left(x_{1}^{0.5}-1\right) \sigma_{1} \mathrm{~d} B_{1}(t)+0.5\left(x_{2}^{0.5}-1\right) \sigma_{2} \mathrm{~d} B_{2}(t) .
\end{aligned}
$$

There exists a constant $N$ such that $f(x)=x^{-0.5}-x^{-1}<N$ on $t>0$; so we can obtain

$\mathrm{d} V$

$$
\begin{aligned}
\leq & {\left[-0.5 k_{1} x_{1}^{1.5}+0.5\left(k_{1}+N \varepsilon_{12}\right) x_{1}\right.} \\
& +0.5\left(r_{1}-\varepsilon_{12}-0.25 \sigma_{1}^{2}\right) x_{1}^{0.5} \\
& \left.-0.5\left(r_{1}-\varepsilon_{12}-0.5 \sigma_{1}^{2}\right)\right] \mathrm{d} t \\
+ & {\left[-0.5 k_{2} x_{2}^{1.5}+0.5\left(k_{2}+N \varepsilon_{21}\right) x_{2}\right.} \\
& +0.5\left(r_{2}-\varepsilon_{21}-0.25 \sigma_{2}^{2}\right) x_{2}^{0.5} \\
& \left.\quad-0.5\left(r_{2}-\varepsilon_{21}-0.5 \sigma_{2}^{2}\right)\right] \mathrm{d} t \\
+ & 0.5\left(x_{1}^{0.5}-1\right) \sigma_{1} \mathrm{~d} B_{1}(t)+0.5\left(x_{2}^{0.5}-1\right) \sigma_{2} \mathrm{~d} B_{2}(t) \\
\leq & M \mathrm{~d} t+0.5\left(x_{1}^{0.5}-1\right) \sigma_{1} \mathrm{~d} B_{1}(t) \\
+ & 0.5\left(x_{2}^{0.5}-1\right) \sigma_{2} \mathrm{~d} B_{2}(t)
\end{aligned}
$$

as long as $\left(x_{1}, x_{2}\right) \in R_{+}^{2}$. Integrating both sides from 0 to $\tau_{k} \wedge$ $T$ and then taking expectations yield

$$
\begin{aligned}
E V( & \left.x_{1}\left(\tau_{k} \wedge T\right), x_{2}\left(\tau_{k} \wedge T\right)\right) \\
& \leq V\left(x_{1}(0), x_{2}(0)\right)+M E\left(\tau_{k} \wedge T\right) \\
& \leq V\left(x_{1}(0), x_{2}(0)\right)+M T .
\end{aligned}
$$

Denote $\Omega_{k}=\left\{\tau_{k} \leq T\right\}$ for $k \geq k_{1}$, by (8), $P\left(\Omega_{k}\right) \geq \varepsilon$. Note that, for every $\omega \in \Omega_{k}$, there is some $i$ such that $x_{i}\left(\tau_{k}, \omega\right)$ equals either $k$ or $1 / k$, and $V\left(x_{1}\left(\tau_{k}, \omega\right), x_{2}\left(\tau_{k}, \omega\right)\right)$ is no less than either $\sqrt{k}-1-0.5 \ln (k)$ or $1 / \sqrt{k}-1-0.5 \ln (1 / k)$. Consequently,

$$
\begin{aligned}
& V\left(x_{1}\left(\tau_{k}, \omega\right), x_{2}\left(\tau_{k}, \omega\right)\right) \\
& \quad \geq[\sqrt{k}-1-0.5 \ln (k)] \wedge\left[\frac{1}{\sqrt{k}}-1-0.5 \ln \left(\frac{1}{k}\right)\right] .
\end{aligned}
$$

It is follows from (13) that

$$
\begin{aligned}
& V\left(x_{1}(0), x_{2}(0)\right)+M T \\
& \quad \geq E\left[I_{\Omega_{k}} V\left(x_{1}\left(\tau_{k}, \omega\right), x_{2}\left(\tau_{k}, \omega\right)\right)\right] \\
& \quad \geq \varepsilon\left([\sqrt{k}-1-0.5 \ln (k)] \wedge\left[\frac{1}{\sqrt{k}}-1-0.5 \ln \left(\frac{1}{k}\right)\right]\right) .
\end{aligned}
$$

Letting $k \rightarrow \infty$ leads to the contradiction

$$
\infty>V\left(x_{1}(0), x_{2}(0)\right)+M T=\infty .
$$

So we must have $\tau_{\infty}=\infty$ a.s.

Theorem 1 shows that the solution of system (4) will remain in the positive cone $R_{+}^{2}$. This nice positive invariant property provides us with a great opportunity to construct different types of the Lyapunov functions to discuss the stationary distribution for system (4) in $R_{+}^{2}$ in more detail.

\section{Stationary Distribution for System (4)}

In order to prove our main results, we require some results in [25], and the technique we used here is motivated by [26-28]. System (4) can be rewritten as

$$
\begin{aligned}
\mathrm{d}\left(\begin{array}{l}
x_{1}(t) \\
x_{2}(t)
\end{array}\right)= & \left(\begin{array}{c}
x_{1}\left(r_{1}-k_{1} x_{1}\right)+\varepsilon_{12}\left(x_{2}-x_{1}\right) \\
x_{2}\left(r_{2}-k_{2} x_{2}\right)+\varepsilon_{21}\left(x_{1}-x_{2}\right)
\end{array}\right) \mathrm{d} t \\
& +\left(\begin{array}{c}
\sigma_{1} x_{1} \\
0
\end{array}\right) \mathrm{d} B_{1}(t)+\left(\begin{array}{c}
0 \\
\sigma_{2} x_{2}
\end{array}\right) \mathrm{d} B_{2}(t) .
\end{aligned}
$$

Its diffusion matrix can be presented as

$$
A\left(x_{1}, x_{2}\right)=\left(\begin{array}{cc}
\sigma_{1}^{2} x_{1}^{2} & 0 \\
0 & \sigma_{2}^{2} x_{2}^{2}
\end{array}\right) .
$$

Assumption B. There exists a bounded domain $R_{+}^{2}$ with regular boundary, having the following properties.

(B1) In the domain $U$ and some neighborhood thereof, the smallest eigenvalue of the diffusion matrix $A(x)$ is bounded away from zero.

(B2) $\sup _{K_{n}} E_{x} \tau<\infty$ for all $n$, where $K_{n}$ is a family of countable compact subsets such that $R_{+}^{2}=\bigcup_{n=1}^{\infty} K_{n}$; $E_{x} \tau$ is the mean time $\tau$ at which a path issuing from $x$ reaches the set $K_{n}$.

Lemma 2 (see [25]). If (B) holds, then the Markov process $X(t)=\left(x_{1}, x_{2}\right)$ has a stable stationary distribution $\mu(\cdot)$ confined on $R_{+}^{2}$.

To validate (B1), it suffices to prove $F$ is uniformly elliptical in $U$, where $F u=b(x) \cdot u_{x}+\left[\operatorname{tr}\left(A(x) u_{x x}\right)\right] / 2$; that is, there is a positive number $M$ such that

$$
\sum_{i, j=1}^{k} a_{i j}(x) \xi_{i} \xi_{j} \geq M|\xi|^{2}, \quad x \in U, \xi \in R^{k}
$$


(see Chapter 3 of [29] and Rayleigh's principle in [30]). To verify (B2), it suffices to show that there exists some neighborhood $U$ and a nonnegative $C^{2}$-function such that, for any $x \in E_{l} \backslash U, L V$ is negative (see[31]).

The deterministic system (2) has two equilibrium points, namely, $E_{0}(0,0)$ and $E^{*}\left(x_{1}^{*}, x_{2}^{*}\right)$. Takeuchi [9] has proved that this single species diffusion model has a positive and globally stable equilibrium point $E^{*}\left(x_{1}^{*}, x_{2}^{*}\right)$ for any diffusion rate; the results obtained in his paper show that no diffusion rate; can change the global stability of the deterministic model.

Suppose $E^{*}\left(x_{1}^{*}, x_{2}^{*}\right)$ is the equilibrium points of system (2). Then, they meet the following equations:

$$
\begin{aligned}
& r_{1}=k_{1} x_{1}^{*}-\varepsilon_{12} \frac{x_{2}^{*}}{x_{1}^{*}}+\varepsilon_{12}, \\
& r_{2}=k_{2} x_{2}^{*}-\varepsilon_{21} \frac{x_{1}^{*}}{x_{2}^{*}}+\varepsilon_{21} .
\end{aligned}
$$

These relations will be useful in the proof of the next theorem. Next, we will show the conditions under which system (4) exists on a stable stationary distribution and discuss the effect of diffusion on the stochastic system.

Theorem 3. Let $\sigma_{1}>0, \sigma_{2}>0$ such that

$$
\begin{gathered}
4 k_{1} k_{2}>\varepsilon_{12} \varepsilon_{21} \beta^{2}, \\
r_{1} \varepsilon_{21} x_{1}^{*}+r_{2} \varepsilon_{12} x_{2}^{*}>\frac{\sigma_{1}^{2}}{2} \varepsilon_{21} x_{1}^{*}+\frac{\sigma_{2}^{2}}{2} \varepsilon_{12} x_{2}^{*} .
\end{gathered}
$$

Then there is a stationary distribution $\mu(\cdot)$ with respect to $R_{+}^{2}$ for system (4) with any initial value $\left(x_{1}(0), x_{2}(0)\right) \in R_{+}^{2}$, where

$$
\beta=\left(\frac{1}{\sqrt{x_{1}^{*}}}-\frac{1}{\sqrt{x_{2}^{*}}}\right)^{2} .
$$

In addition, condition (22) can be satisfied when

$$
r_{1}>\frac{\sigma_{1}^{2}}{2}, \quad r_{2}>\frac{\sigma_{2}^{2}}{2} \text {. }
$$

Proof. Define $V: R_{+}^{2} \rightarrow R_{+}$,

$$
\begin{aligned}
V\left(x_{1}, x_{2}\right)= & \left(x_{1}-x_{1}^{*}-x_{1}^{*} \ln \frac{x_{1}}{x_{1}^{*}}\right) \\
& +k\left(x_{2}-x_{2}^{*}-x_{2}^{*} \ln \frac{x_{2}}{x_{2}^{*}}\right)=V_{1}+k V_{2},
\end{aligned}
$$

where $k$ is a positive constant to be determined later. $V\left(x_{1}, x_{2}\right)$ is a positive definite function for all $\left(x_{1}, x_{2}\right) \neq\left(x_{1}^{*}, x_{2}^{*}\right)$. By Itô's formula, we can calculate

$$
\begin{aligned}
& \mathrm{d} V_{1}\left(x_{1}, x_{2}\right) \\
& =\left(1-\frac{x_{1}^{*}}{x_{1}}\right) \mathrm{d} x_{1}+\frac{1}{2} \frac{x_{1}^{*}}{x_{1}^{2}} \sigma_{1}^{2} x_{1}^{2} \mathrm{~d} t \\
& =\left(x_{1}-x_{1}^{*}\right)\left[\left(r_{1}-k_{1} x_{1}+\varepsilon_{12} \frac{x_{2}}{x_{1}}-\varepsilon_{12}\right) \mathrm{d} t\right. \\
& \left.+\sigma_{1} \mathrm{~d} B_{1}(t)\right]+\frac{1}{2} x_{1}^{*} \sigma_{1}^{2} \mathrm{~d} t
\end{aligned}
$$

$$
\begin{aligned}
& =\left(x_{1}-x_{1}^{*}\right)\left[k_{1} x_{1}^{*}-\varepsilon_{12} \frac{x_{2}^{*}}{x_{1}^{*}}+\varepsilon_{12}\right. \\
& \left.\quad-k_{1} x_{1}+\varepsilon_{12} \frac{x_{2}}{x_{1}}-\varepsilon_{12}\right] \mathrm{d} t \\
& +\frac{1}{2} x_{1}^{*} \sigma_{1}^{2} \mathrm{~d} t+\sigma_{1}\left(x_{1}-x_{1}^{*}\right) \mathrm{d} B_{1}(t) \\
& =\left(x_{1}-x_{1}^{*}\right)\left[k_{1}\left(x_{1}^{*}-x_{1}\right)+\varepsilon_{12}\left(\frac{x_{2}}{x_{1}}-\frac{x_{2}^{*}}{x_{1}^{*}}\right)\right] \mathrm{d} t \\
& +\frac{1}{2} x_{1}^{*} \sigma_{1}^{2} \mathrm{~d} t+\sigma_{1}\left(x_{1}-x_{1}^{*}\right) \mathrm{d} B_{1}(t) \\
& =\left[-k_{1}\left(x_{1}-x_{1}^{*}\right)^{2}+\varepsilon_{12}\left(x_{1}-x_{1}^{*}\right)\left(\frac{x_{2}}{x_{1}}-\frac{x_{2}^{*}}{x_{1}^{*}}\right)\right. \\
& \left.\quad+\frac{1}{2} x_{1}^{*} \sigma_{1}^{2}\right] \mathrm{d} t+\sigma_{1}\left(x_{1}-x_{1}^{*}\right) \mathrm{d} B_{1}(t) \\
& =L V_{1}\left(x_{1}, x_{2}\right) \mathrm{d} t+\sigma_{1}\left(x_{1}-x_{1}^{*}\right) \mathrm{d} B_{1}(t),
\end{aligned}
$$$$
\mathrm{d} V_{2}\left(x_{1}, x_{2}\right)
$$$$
=\left(1-\frac{x_{2}^{*}}{x_{2}}\right) \mathrm{d} x_{2}+\frac{1}{2} \frac{x_{2}^{*}}{x_{2}^{2}} \sigma_{2}^{2} x_{2}^{2} \mathrm{~d} t
$$$$
=\left(x_{2}-x_{2}^{*}\right)\left[\left(r_{2}-k_{2} x_{2}+\varepsilon_{21} \frac{x_{1}}{x_{2}}-\varepsilon_{21}\right) \mathrm{d} t\right.
$$$$
\left.+\sigma_{2} \mathrm{~d} B_{2}(t)\right]+\frac{1}{2} \sigma_{2}^{2} x_{2}^{*} \mathrm{~d} t
$$$$
=\left(x_{2}-x_{2}^{*}\right)\left[k_{2} x_{2}^{*}-\varepsilon_{21} \frac{x_{1}^{*}}{x_{2}^{*}}+\varepsilon_{21}-k_{2} x_{2}\right.
$$$$
\left.+\varepsilon_{21} \frac{x_{1}}{x_{2}}-\varepsilon_{21}\right] \mathrm{d} t
$$$$
+\frac{1}{2} \sigma_{2}^{2} x_{2}^{*} \mathrm{~d} t+\sigma_{2}\left(x_{2}-x_{2}^{*}\right) \mathrm{d} B_{2}(t)
$$$$
=\left(x_{2}-x_{2}^{*}\right)\left[k_{2}\left(x_{2}^{*}-x_{2}\right)+\varepsilon_{21}\left(\frac{x_{1}}{x_{2}}-\frac{x_{1}^{*}}{x_{2}^{*}}\right)\right] \mathrm{d} t
$$$$
+\frac{1}{2} \sigma_{2}^{2} x_{2}^{*} \mathrm{~d} t+\sigma_{2}\left(x_{2}-x_{2}^{*}\right) \mathrm{d} B_{2}(t)
$$$$
=\left[-k_{2}\left(x_{2}-x_{2}^{*}\right)^{2}+\varepsilon_{21}\left(x_{2}-x_{2}^{*}\right)\left(\frac{x_{1}}{x_{2}}-\frac{x_{1}^{*}}{x_{2}^{*}}\right)\right.
$$$$
\left.+\frac{1}{2} \sigma_{2}^{2} x_{2}^{*}\right] \mathrm{d} t+\sigma_{2}\left(x_{2}-x_{2}^{*}\right) \mathrm{d} B_{2}(t)
$$$$
=L V_{2}\left(x_{1}, x_{2}\right) \mathrm{d} t+\sigma_{2}\left(x_{2}-x_{2}^{*}\right) \mathrm{d} B_{2}(t) \text {. }
$$

Then we have

$$
\begin{aligned}
\mathrm{d} V\left(x_{1}, x_{2}\right)= & \mathrm{d} V_{1}\left(x_{1}, x_{2}\right)+k \mathrm{~d} V_{2}\left(x_{1}, x_{2}\right) \\
= & L V\left(x_{1}, x_{2}\right) \mathrm{d} t+\sigma_{1}\left(x_{1}-x_{1}^{*}\right) \mathrm{d} B_{1}(t) \\
& +\sigma_{2}\left(x_{2}-x_{2}^{*}\right) \mathrm{d} B_{2}(t) .
\end{aligned}
$$


Choosing $k=\varepsilon_{12} / \varepsilon_{21}$, we can obtain

$$
\begin{aligned}
& L V\left(x_{1}, x_{2}\right) \\
& =-k_{1}\left(x_{1}-x_{1}^{*}\right)^{2}-k_{2} \frac{\varepsilon_{12}}{\varepsilon_{21}}\left(x_{2}-x_{2}^{*}\right)^{2} \\
& \quad+\frac{1}{2} x_{1}^{*} \sigma_{1}^{2}+\frac{1}{2} \frac{\varepsilon_{12}}{\varepsilon_{21}} \sigma_{2}^{2} x_{2}^{*}+\varepsilon_{12} \\
& \quad \times\left[\left(x_{1}-x_{1}^{*}\right)\left(\frac{x_{2}}{x_{1}}-\frac{x_{2}^{*}}{x_{1}^{*}}\right)+\left(x_{2}-x_{2}^{*}\right)\left(\frac{x_{1}}{x_{2}}-\frac{x_{1}^{*}}{x_{2}^{*}}\right)\right] \\
& \quad-k_{1}\left(x_{1}-x_{1}^{*}\right)^{2}-k_{2} \frac{\varepsilon_{12}}{\varepsilon_{21}}\left(x_{2}-x_{2}^{*}\right)^{2} \\
& \quad+\sigma-\varepsilon_{12} \Gamma\left(x_{1}, x_{2}\right),
\end{aligned}
$$

where

$$
\begin{aligned}
& \sigma=\frac{1}{2} x_{1}^{*} \sigma_{1}^{2}+\frac{1}{2} \frac{\varepsilon_{12}}{\varepsilon_{21}} \sigma_{2}^{2} x_{2}^{*}, \\
& \Gamma\left(x_{1}, x_{2}\right) \\
& =-\left[\left(x_{1}-x_{1}^{*}\right)\left(\frac{x_{2}}{x_{1}}-\frac{x_{2}^{*}}{x_{1}^{*}}\right)+\left(x_{2}-x_{2}^{*}\right)\left(\frac{x_{1}}{x_{2}}-\frac{x_{1}^{*}}{x_{2}^{*}}\right)\right] \\
& =-\frac{\left(x_{1}-x_{1}^{*}\right)\left(x_{1}^{*} x_{2}-x_{1} x_{2}^{*}\right)}{x_{1} x_{1}^{*}}-\frac{\left(x_{2}-x_{2}^{*}\right)\left(x_{1} x_{2}^{*}-x_{2} x_{1}^{*}\right)}{x_{2} x_{2}^{*}} \\
& =-\frac{\left(x_{1}-x_{1}^{*}\right)\left(x_{2}\left(x_{1}^{*}-x_{1}\right)+x_{1}\left(x_{2}-x_{2}^{*}\right)\right)}{x_{1} x_{1}^{*}} \\
& -\frac{\left(x_{2}-x_{2}^{*}\right)\left(x_{1}\left(x_{2}^{*}-x_{2}\right)+x_{2}\left(x_{1}-x_{1}^{*}\right)\right)}{x_{2} x_{2}^{*}} \\
& =\frac{x_{2}\left(x_{1}-x_{1}^{*}\right)^{2}}{x_{1} x_{1}^{*}}+\frac{x_{1}\left(x_{2}-x_{2}^{*}\right)^{2}}{x_{2} x_{2}^{*}} \\
& -\left(\frac{1}{x_{1}^{*}}+\frac{1}{x_{2}^{*}}\right)\left(x_{1}-x_{1}^{*}\right)\left(x_{2}-x_{2}^{*}\right) \\
& \geq\left(2 \sqrt{\frac{1}{x_{1}^{*} x_{2}^{*}}}-\frac{1}{x_{1}^{*}}-\frac{1}{x_{2}^{*}}\right)\left(x_{1}-x_{1}^{*}\right)\left(x_{2}-x_{2}^{*}\right) \\
& =-\left(\frac{1}{\sqrt{x_{1}^{*}}}-\frac{1}{\sqrt{x_{2}^{*}}}\right)^{2}\left(x_{1}-x_{1}^{*}\right)\left(x_{2}-x_{2}^{*}\right) \text {. }
\end{aligned}
$$

Then we have

$$
\begin{aligned}
L V \leq & -k_{1}\left(x_{1}-x_{1}^{*}\right)^{2}-k_{2} \frac{\varepsilon_{12}}{\varepsilon_{21}}\left(x_{2}-x_{2}^{*}\right)^{2} \\
& +\sigma+\varepsilon_{12}\left(\frac{1}{\sqrt{x_{1}^{*}}}-\frac{1}{\sqrt{x_{2}^{*}}}\right)^{2}\left(x_{1}-x_{1}^{*}\right) \\
& \times\left(x_{2}-x_{2}^{*}\right) \triangleq R\left(x_{1}, x_{2}\right) .
\end{aligned}
$$

If we denote $\bar{x}_{1}=x_{1}-x_{1}^{*}, \bar{x}_{2}=x_{2}-x_{2}^{*}$, then

$$
\begin{aligned}
L V \leq & -k_{1} \bar{x}_{1}^{2}-k_{2} \frac{\varepsilon_{12}}{\varepsilon_{21}} \bar{x}_{2}^{2}+\sigma \\
& +\varepsilon_{12}\left(\frac{1}{\sqrt{x_{1}^{*}}}-\frac{1}{\sqrt{x_{2}^{*}}}\right)^{2} \bar{x}_{1} \bar{x}_{2} \triangleq G\left(\bar{x}_{1}, \bar{x}_{2}\right) .
\end{aligned}
$$

It is well known that $G\left(\bar{x}_{1}, \bar{x}_{2}\right)=0$ is an elliptic-curve when

$$
I_{2}=\left|\begin{array}{cc}
k_{1} & \frac{-\varepsilon_{12} \beta}{2} \\
\frac{-\varepsilon_{12} \beta}{2} & k_{2} \frac{\varepsilon_{12}}{\varepsilon_{21}}
\end{array}\right|>0,
$$

that is,

$$
4 k_{1} k_{2}>\varepsilon_{12} \varepsilon_{21} \beta^{2} .
$$

Now we take $U$ to be the intersection of $\left(G\left(\bar{x}_{1}, \bar{x}_{2}\right) \geq 0\right)$ and $R_{+}^{2}$ with $U \subseteq R_{+}^{2}$. So, for $\left(x_{1}, x_{2}\right) \in R_{+}^{2} / U, L V$ is negative, which implies condition (B2) is satisfied. Besides, If $U$ is bounded away from $(0,0)$, that is,

$$
\lim _{x_{1}, x_{2} \rightarrow 0} L V\left(x_{1}, x_{2}\right)=-k_{1}\left(x_{1}^{*}\right)^{2}-k_{2} \frac{\varepsilon_{12}}{\varepsilon_{21}}\left(x_{2}^{*}\right)^{2}+\sigma<0,
$$

which can be satisfied when

$$
\begin{aligned}
& k_{1} \varepsilon_{21}\left(x_{1}^{*}\right)^{2}+k_{2} \varepsilon_{12}\left(x_{2}^{*}\right)^{2} \\
& \quad=r_{1} \varepsilon_{21} x_{1}^{*}+r_{2} \varepsilon_{12} x_{2}^{*}>\frac{\sigma_{1}^{2}}{2} \varepsilon_{21} x_{1}^{*}+\frac{\sigma_{2}^{2}}{2} \varepsilon_{12} x_{2}^{*},
\end{aligned}
$$

then there is a constant $M>0$ such that

$$
\begin{aligned}
& \sum_{i, j=1}^{2} a_{i j}\left(x_{1}, x_{2}\right) \xi_{i} \xi_{j} \\
& \quad=\sigma_{1}^{2} x_{1}^{2} \xi_{1}^{2}+\sigma_{2}^{2} x_{2}^{2} \xi_{2}^{2} \geq M|\xi|^{2} \quad \forall\left(x_{1}, x_{2}\right) \in U, \xi \in R^{2},
\end{aligned}
$$

which implies condition (B1) is also satisfied. Therefore, system (4) has a stable stationary distribution $\mu(\cdot)$ confined on $R_{+}^{2}$. These together with the positive invariant property of $R_{+}^{2}$ complete our proof.

Lemma 4 (see [32, Corollary 1]). Equation $d x(t)=x(t)(r-$ $k x(t)) d t+\alpha x(t) d B(t)$ has a nontrivial stationary distribution if and only if $\alpha^{2}<2 r$.

Remark 5. Suppose there is no diffusion; that is, $\varepsilon_{12}=$ $\varepsilon_{21}=0$. Then condition (21) is always satisfied, and the corresponding equations,

$$
\begin{aligned}
& \mathrm{d} x_{1}=x_{1}\left(r_{1}-k_{1} x_{1}\right) \mathrm{d} t+\sigma_{1} x_{1} \mathrm{~d} B_{1}(t), \\
& \mathrm{d} x_{2}=x_{2}\left(r_{2}-k_{2} x_{2}\right) \mathrm{d} t+\sigma_{2} x_{2} \mathrm{~d} B_{2}(t),
\end{aligned}
$$

have stationary distributions when $r_{1}>\sigma_{1}^{2} / 2, r_{2}>\sigma_{2}^{2} / 2$. This is in agreement with the results in the literature [32] (see Lemma 4). 
Remark 6. Suppose condition (24) is satisfied. Then the species has a nontrivial stationary distribution in all patches if the patches are isolated; that is, the diffusion among patches is neglected, and the species is confined to each patch. Condition (21) can be satisfied when we choose $\varepsilon_{12}, \varepsilon_{21}$ sufficiently small. Then we have a conclusion that diffusion cannot change the existence of stable stationary distribution for stochastic system if the strength of diffusion rate is small enough.

Remark 7. An immediate consequence of condition (22) is that environmental noises are against the stationary distribution for stochastic system. If $r_{1}<\sigma_{1}^{2} / 2, r_{2}>\sigma_{2}^{2} / 2$; that is, only species in the 2 nd patch have a nontrivial stationary distribution when there is no diffusion. But we can choose $\varepsilon_{21}$ sufficiently small such that conditions in Theorem 3 are satisfied, and there exists a stationary distribution for $\left(x_{1}(t), x_{2}(t)\right)$. This implies small diffusion rate has some stabilizing effects on stochastic system. However, if we choose $\varepsilon_{21}$ sufficiently large, then the conditions of Theorem 3 are destroyed which implies large diffusion rate also has some destabilizing effects on stochastic models.

\section{Examples and Numerical Simulation}

Now we will give three examples to explain both the stabilizing and destabilizing effects of diffusion on the population dynamics. The data we used here are only some hypothetical data which are used to explain the effect of diffusion. We use the Milsteins Higher Order Method mentioned in [33] to numerically simulate (4):

$$
\begin{aligned}
x_{1}^{k+1}= & x_{1}^{k}+\left[x_{1}^{k}\left(r_{1}-k_{1} x_{1}^{k}\right)+\varepsilon_{12}\left(x_{2}^{k}-x_{1}^{k}\right)\right] \Delta t \\
& +\sigma_{1} x_{1}^{k} \sqrt{\Delta t} \xi_{k}+\frac{\sigma_{1}^{2}}{2} x_{1}^{k}\left(\Delta t \xi_{k}^{2}-\Delta t\right), \\
x_{2}^{k+1}= & x_{2}^{k}+\left[x_{2}^{k}\left(r_{2}-k_{2} x_{2}^{k}\right)+\varepsilon_{21}\left(x_{1}^{k}-x_{2}^{k}\right)\right] \Delta t \\
& +\sigma_{2} x_{2}^{k} \sqrt{\Delta t} \eta_{k}+\frac{\sigma_{2}^{2}}{2} x_{2}^{k}\left(\Delta t \eta_{k}^{2}-\Delta t\right),
\end{aligned}
$$

where $\xi_{k}$ and $\eta_{k}$ are the Gaussian random variables $N(0,1)$.

It is very difficult to choose parameters in the system from realistic estimation. The estimation of the parameters can be derived by some statistical methods and filtering theory which are linked to statistical problems and filtering problems. Therefore, we will only use some hypothetical parameters to verify the theoretical effects in this section.

Example 8. For system (38), we let $r_{1}=0.9, k_{1}=1.2, \sigma_{1}=$ $\sqrt{2}, r_{2}=1.1, k_{2}=0.2$, and $\sigma_{2}=0.8$. Note that $r_{1}<\sigma_{1}^{2} / 2$ and $r_{2}>\sigma_{2}^{2} / 2$; so for system (38), species in the 1st patch has a Dirac delta distribution with mass concentrated in 0 , and species in the 2 nd patch has a nontrivial distribution. (see literature [32].) Numerical simulations of (38) are showed in Figures 1(a) and 1(b).

Example 9. For system (4), we let $r_{1}=0.9, k_{1}=1.2, \varepsilon_{12}=$ $0.1, \sigma_{1}=\sqrt{2}, r_{2}=1.1, k_{2}=0.2, \varepsilon_{21}=0.4$, and $\sigma_{2}=$

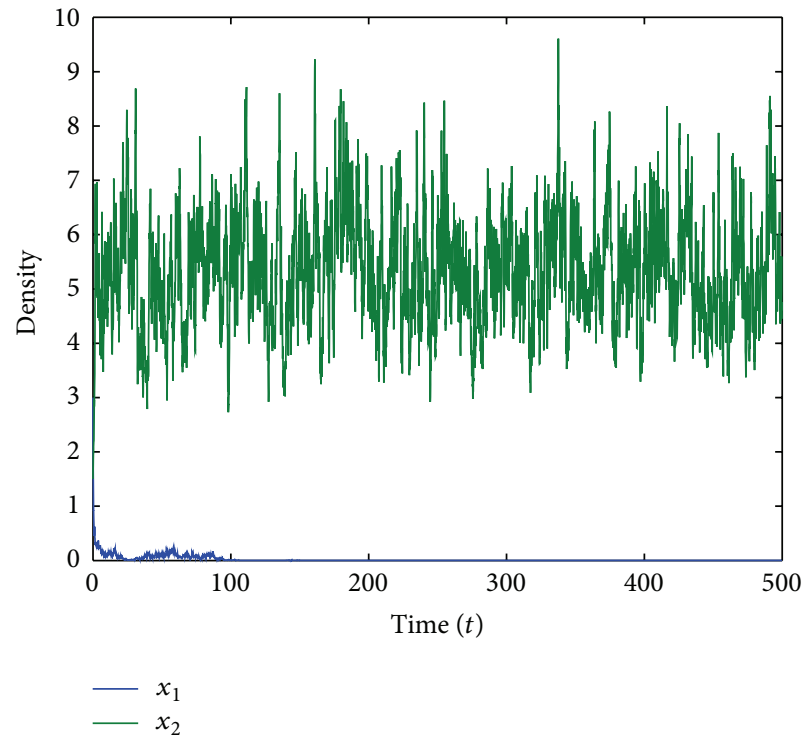

(a)

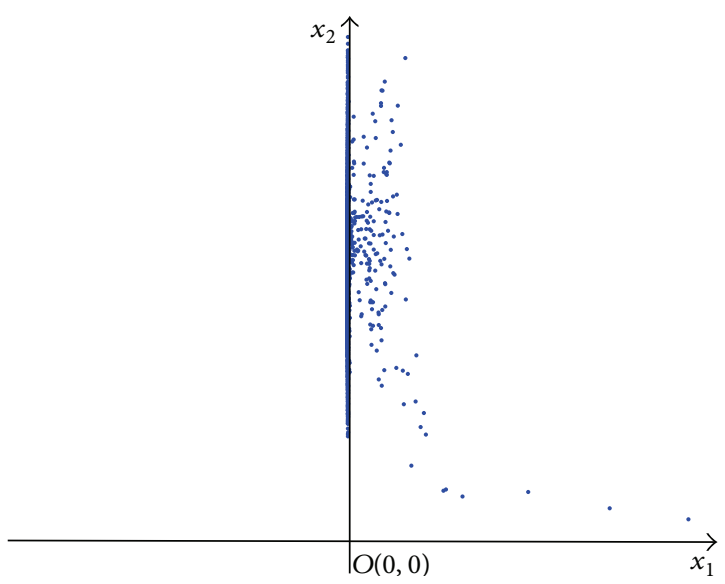

(b)

Figure 1: Numerical simulations of Example 8 (there is no diffusion). Species in the 1st patch has a Dirac delta distribution with mass concentrated in 0 , and species in the 2 nd patch has a stationary distribution.

0.8 . Its corresponding deterministic system (2) has a globally asymptotically stable equilibrium point $E^{*}\left(x^{*}, y^{*}\right)=(1,4)$. We also have

$$
\begin{gathered}
\sigma_{1}=\sqrt{2}>0, \quad \sigma_{2}=0.8>0, \\
\beta=\left(\frac{1}{\sqrt{x_{1}^{*}}}-\frac{1}{\sqrt{x_{2}^{*}}}\right)^{2}=\frac{1}{4}, \\
4 k_{1} k_{2}=0.96>0.0025=\varepsilon_{12} \varepsilon_{21} \beta^{2}, \\
r_{1} \varepsilon_{21} x_{1}^{*}+r_{2} \varepsilon_{12} x_{2}^{*}=0.8>0.528=\frac{\sigma_{1}^{2}}{2} \varepsilon_{21} x_{1}^{*}+\frac{\sigma_{2}^{2}}{2} \varepsilon_{12} x_{2}^{*} .
\end{gathered}
$$



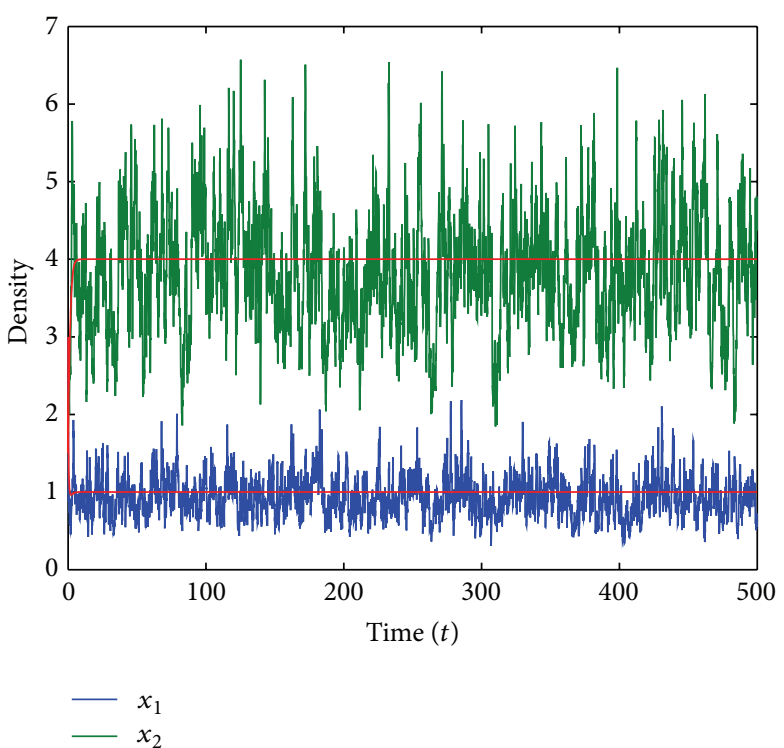

(a)

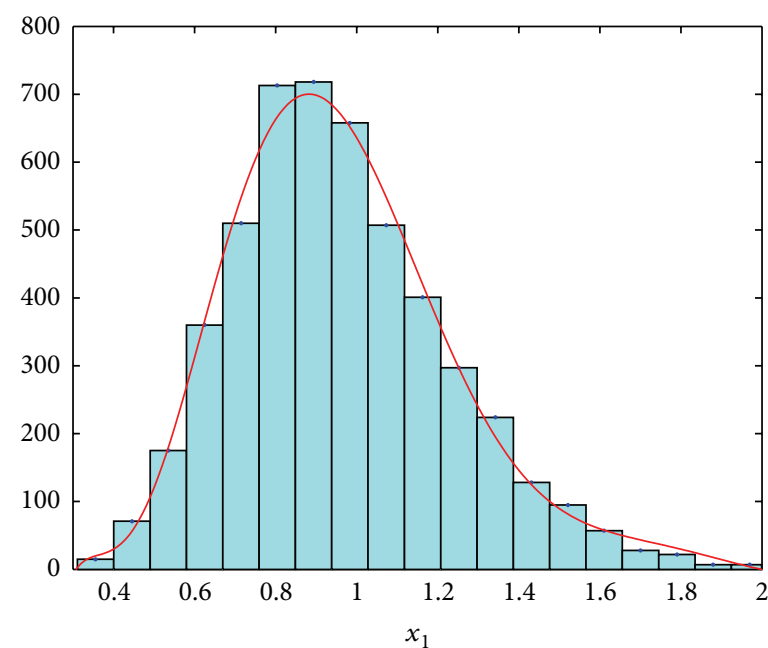

(c)

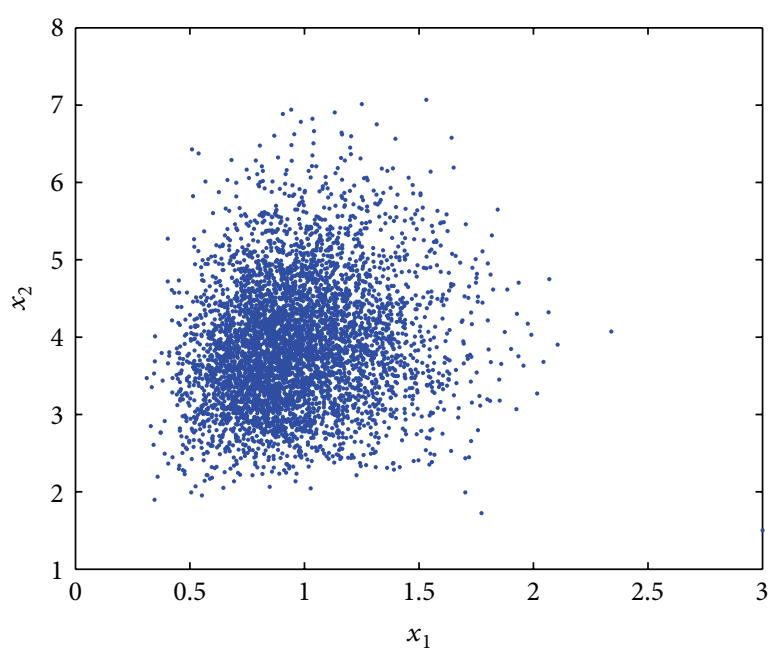

(b)

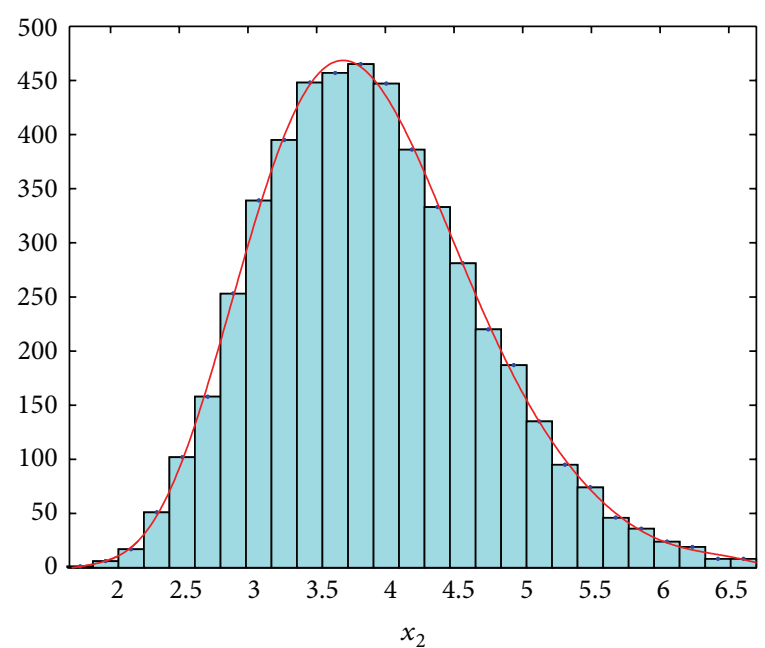

(d)

FIGURE 2: Numerical simulations of Example 9. In this example, we choose $\varepsilon_{21}$ sufficiently small such that conditions in Theorem 3 are satisfied, and there is a stationary distribution for $\left(x_{1}(t), x_{2}(t)\right)$; (c) and (d) are distributions of $x_{1}$ and $x_{2}$, respectively.

So, from Theorem 3, we obtain that there is a stationary distribution $\mu(\cdot)$ with respect to $R_{+}^{2}$ for system (4) with initial value $\left(x_{1}(0), x_{2}(0)\right) \in R_{+}^{2}$ (see Figures $2(\mathrm{a}), 2(\mathrm{~b}), 2(\mathrm{c})$, and $2(\mathrm{~d})$ ). The stabilizing effect of small diffusion rate can be seen clearly from this example.

Example 10. For system (4), we let $r_{1}=0.9, k_{1}=1.2, \varepsilon_{12}=$ $0.1, \sigma_{1}=\sqrt{2}, r_{2}=1.1, k_{2}=0.2, \varepsilon_{21}=5$, and $\sigma_{2}=0.8$. It is clear that conditions of Theorem 3 are destroyed and numerical simulations of this example showed in Figures 3(a) and $3(\mathrm{~b})$. The destabilizing effect of large diffusion rate can be seen clearly from this example.

\section{Concluding Remarks}

The main objective of this paper is to study the effects of dispersal on stationary distribution for a stochastic logistic diffusion system. We show that the dispersal stabilizes the system when the dispersal rate is small, and destabilizes the system, when the dispersal rate is large. Our results show that small dispersal rate cannot change the existence of stationary distribution for the stochastic model such as it cannot change the global stability of the deterministic model. Though diffusions have stabilizing effects, our examples show that dispersal may also have the side effects which 


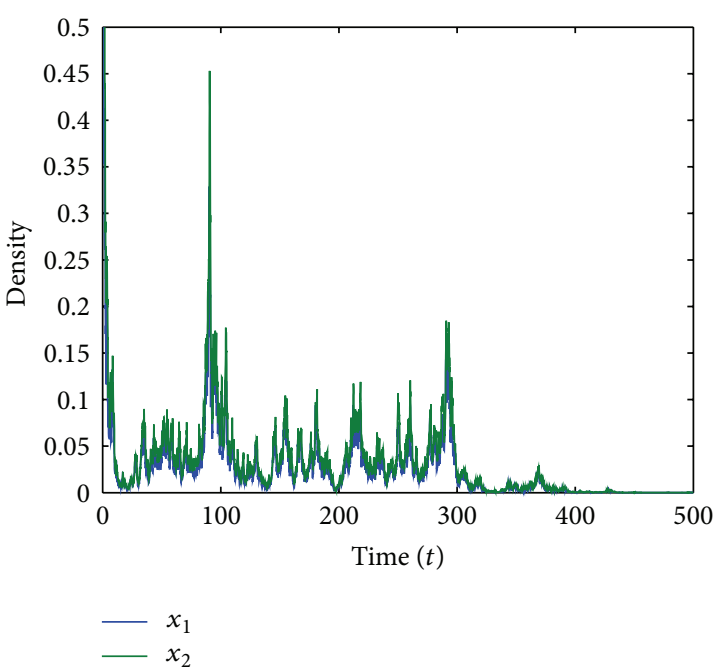

(a)

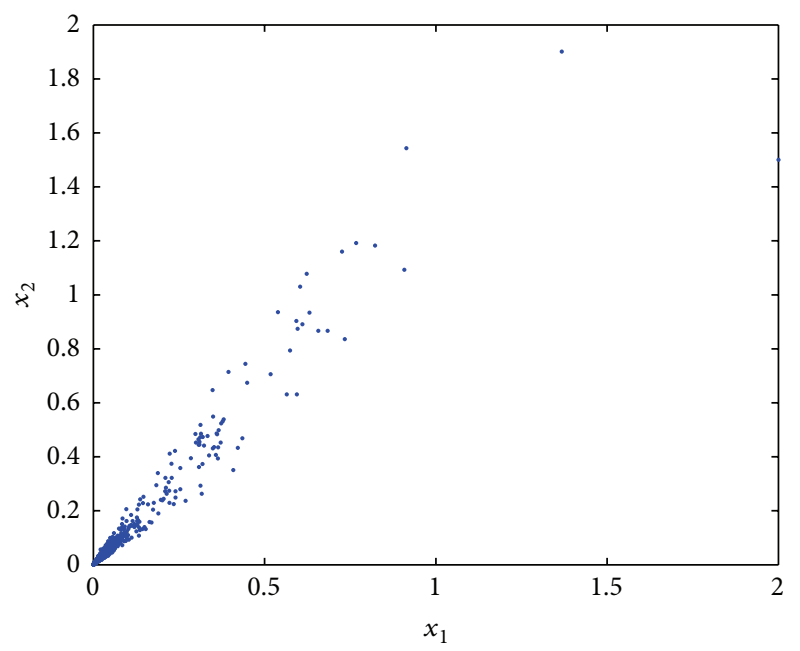

(b)

Figure 3: Numerical simulations of Example 10. In this example, we choose $\varepsilon_{21}$ sufficiently large such that conditions in Theorem 3 are destroyed which show the destabilizing effect of large diffusion rate.

result in destabilization. This suggests that dispersal among patches should be regulated. Their ecological implications are that neither no diffusion nor unlimited diffusion may serve the interest of stabilizing the given ecosystem in random environments! This observation may be useful in planning and controlling of ecosystems.

\section{Acknowledgments}

The authors would like to thank the editor and the referees for their suggestions which improved the presentation of this paper. This paper was partially supported by Grants from the National Natural Science Foundation of China (no. 11171081), (no. 11171056), (no. 11126219), (no. 11001032), and (no. 11101183), Shandong Provincial Natural Science Foundation of China (Grant no. ZR2011AM004), and Natural
Scientific Research Innovation Foundation in Harbin Institute of Technology (HIT.NSRIF.2011094).

\section{References}

[1] J. G. Skellam, "Random dispersal in theoretical populations," Biometrika, vol. 38, pp. 196-218, 1951.

[2] A. Hastings, "Dynamics of a single species in a spatially varying environment: the stabilizing role of high dispersal rates," Journal of Mathematical Biology, vol. 16, no. 1, pp. 49-55, 1982.

[3] H. I. Freedman, B. Rai, and P. Waltman, "Mathematical models of population interactions with dispersal. II. Differential survival in a change of habitat," Journal of Mathematical Analysis and Applications, vol. 115, no. 1, pp. 140-154, 1986.

[4] L. J. S. Allen, "Persistence, extinction, and critical patch number for island populations," Journal of Mathematical Biology, vol. 24, no. 6, pp. 617-625, 1987.

[5] E. Beretta and Y. Takeuchi, "Global stability of single-species diffusion Volterra models with continuous time delays," Bulletin of Mathematical Biology, vol. 49, no. 4, pp. 431-448, 1987.

[6] E. Beretta and Y. Takeuchi, "Global asymptotic stability of Lotka-Volterra diffusion models with continuous time delay," SIAM Journal on Applied Mathematics, vol. 48, no. 3, pp. 627651, 1988.

[7] W. Wang and L. Chen, "Global stability of a population dispersal in a two-patch environment," Dynamic Systems and Applications, vol. 6, no. 2, pp. 207-215, 1997.

[8] J. Cui, Y. Takeuchi, and Z. Lin, "Permanence and extinction for dispersal population systems," Journal of Mathematical Analysis and Applications, vol. 298, no. 1, pp. 73-93, 2004.

[9] Y. Takeuchi, "Cooperative systems theory and global stability of diffusion models," Acta Applicandae Mathematicae, vol. 14, no. 1-2, pp. 49-57, 1989.

[10] S. A. Levin, "Dispersion and population interactions," The American Naturalist, vol. 108, no. 960, pp. 207-228, 1974.

[11] S. A. Levin, "Spatial patterning and the structure of ecological communities," in Some Mathematical Questions in Biology, vol. 8 of Lectures on Mathematics in the Life Sciences, pp. 1-35, American Mathematical Society, Providence, RI, USA, 1976.

[12] H. I. Freedman and Y. Takeuchi, "Global stability and predator dynamics in a model of prey dispersal in a patchy environment," Nonlinear Analysis. Theory, Methods \& Applications, vol. 13, no. 8, pp. 993-1002, 1989.

[13] Y. Kuang and Y. Takeuchi, "Predator-prey dynamics in models of prey dispersal in two-patch environments," Mathematical Biosciences, vol. 120, no. 1, pp. 77-98, 1994.

[14] Y. Takeuchi, "Diffusion-mediated persistence in two-species competition Lotka-Volterra model," Mathematical Biosciences, vol. 95, no. 1, pp. 65-83, 1989.

[15] J. Li and J. Yan, "Permanence and extinction for a nonlinear diffusive predator-prey system," Nonlinear Analysis. Theory, Methods \& Applications, vol. 71, no. 1-2, pp. 399-417, 2009.

[16] Y. Takeuchi and Z. Y. Lu, "Permanence and global stability for competitive Lotka-Volterra diffusion systems," Nonlinear Analysis. Theory, Methods \& Applications, vol. 24, no. 1, pp. 91104, 1995.

[17] T. C. Gard, "Persistence in stochastic food web models," Bulletin of Mathematical Biology, vol. 46, no. 3, pp. 357-370, 1984.

[18] T. C. Gard, "Stability for multispecies population models in random environments," Nonlinear Analysis. Theory, Methods \& Applications, vol. 10, no. 12, pp. 1411-1419, 1986. 
[19] R. M. May, Stability and Complexity in Model Ecosystems, Princeton University, Princeton, NJ, USA, 1973.

[20] M. Fan and K. Wang, "Study on harvested population with diffusional migration," Journal of Systems Science and Complexity, vol. 14, no. 2, pp. 139-148, 2001.

[21] X. Zou and K. Wang, "The protection zone of biological population," Nonlinear Analysis. Real World Applications, vol. 12, no. 2, pp. 956-964, 2011.

[22] X. Mao, G. Marion, and E. Renshaw, "Environmental Brownian noise suppresses explosions in population dynamics," Stochastic Processes and Their Applications, vol. 97, no. 1, pp. 95-110, 2002.

[23] L. Arnold, Stochastic Differential Equations: Theory and Applications, John Wiley \& Sons, New York, NY, USA, 1972.

[24] A. Friedman, Stochastic Differential Equations and Applications. Vol. 2. Probability and Mathematical Statistics, vol. 28, Academic Press, New York, NY, USA, 1976.

[25] R. Z. Hasminskiǔ, Stochastic Stability of Differential Equations, vol. 7 of Monographs and Textbooks on Mechanics of Solids and Fluids: Mechanics and Analysis, Sijthoff \& Noordhoff, Alphen aan den Rijn, The Netherlands, 1980.

[26] C. Ji, D. Jiang, and N. Shi, "A note on a predator-prey model with modified Leslie-Gower and Holling-type II schemes with stochastic perturbation," Journal of Mathematical Analysis and Applications, vol. 377, no. 1, pp. 435-440, 2011.

[27] C. Ji and D. Jiang, "Dynamics of a stochastic density dependent predator-prey system with Beddington-DeAngelis functional response," Journal of Mathematical Analysis and Applications, vol. 381, no. 1, pp. 441-453, 2011.

[28] X. Mao, "Stationary distribution of stochastic population systems," Systems \& Control Letters, vol. 60, no. 6, pp. 398-405, 2011.

[29] T. C. Gard, Introduction to Stochastic Differential Equations, vol. 270, Madison Avenue, New York, NY, USA, 1988.

[30] G. Strang, Linear Algebra and Its Applications, Harcourt Brace, Watkins, Minn, USA, 3rd edition, 1988.

[31] C. Zhu and G. Yin, "Asymptotic properties of hybrid diffusion systems," SIAM Journal on Control and Optimization, vol. 46, no. 4, pp. 1155-1179, 2007.

[32] S. Pasquali, "The stochastic logistic equation: stationary solutions and their stability," Rendiconti del Seminario Matematico della Università di Padova, vol. 106, pp. 165-183, 2001.

[33] D. J. Higham, "An algorithmic introduction to numerical simulation of stochastic differential equations," SIAM Review, vol. 43, no. 3, pp. 525-546, 2001. 


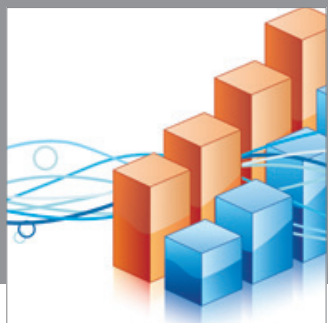

Advances in

Operations Research

mansans

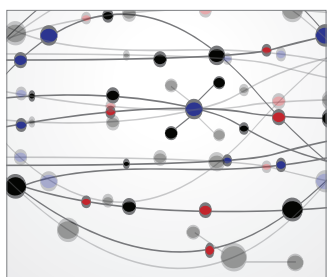

The Scientific World Journal
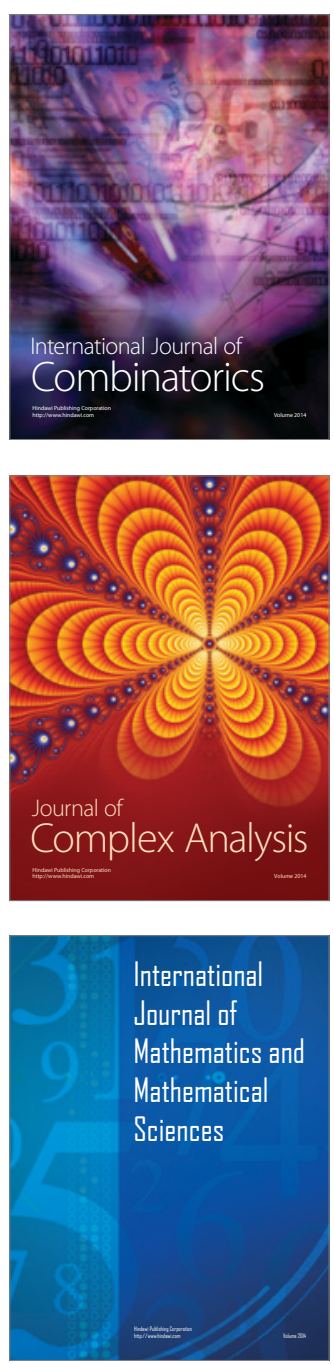
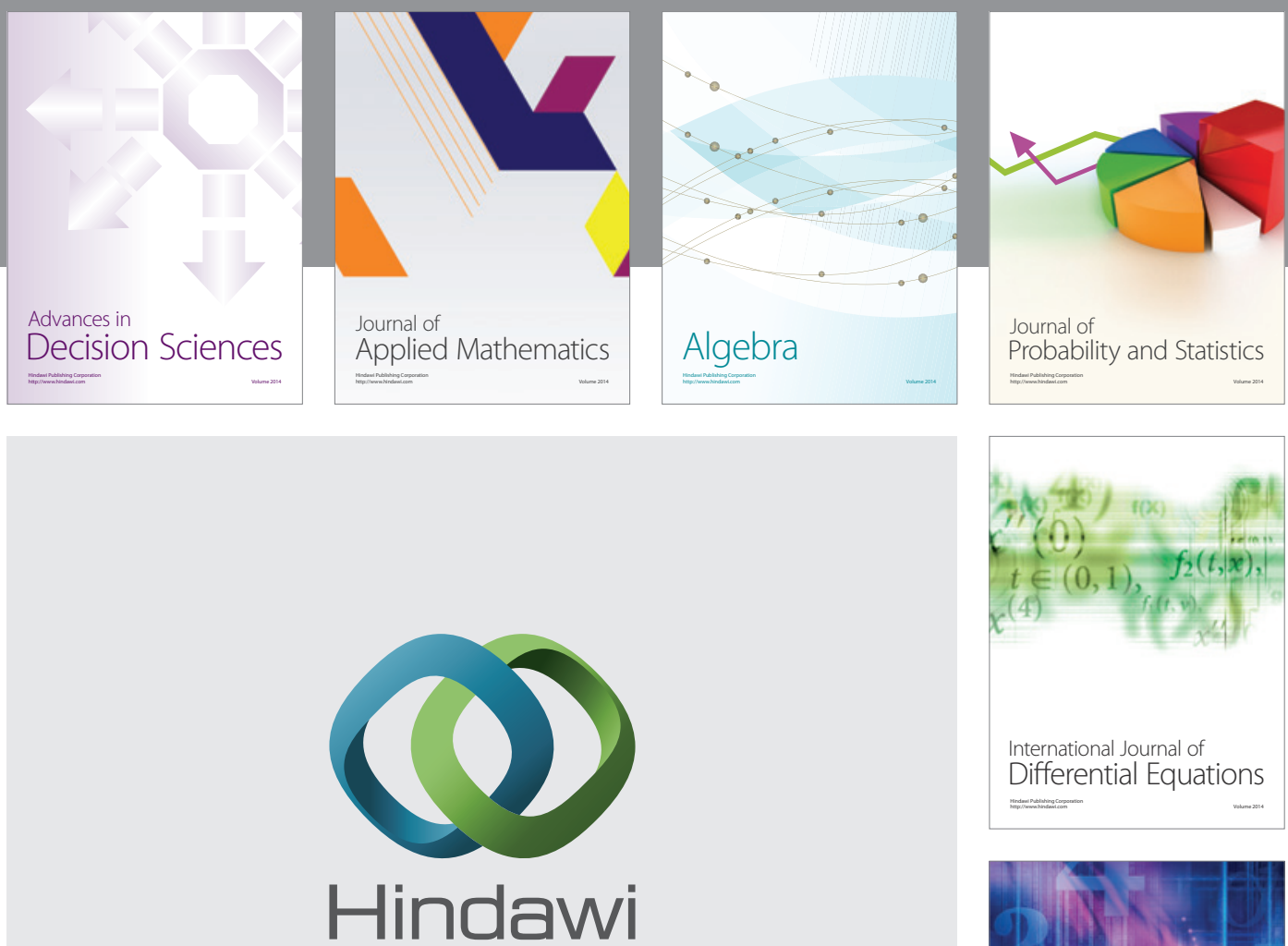

Submit your manuscripts at http://www.hindawi.com
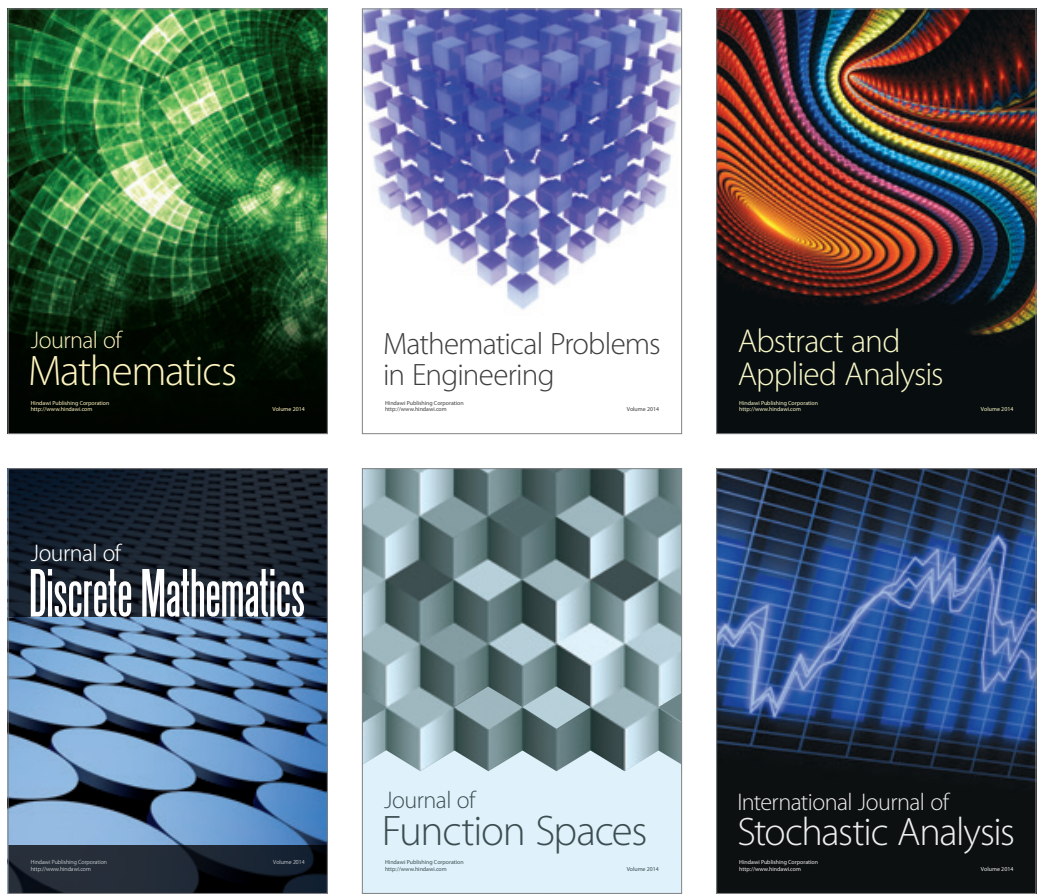

Journal of

Function Spaces

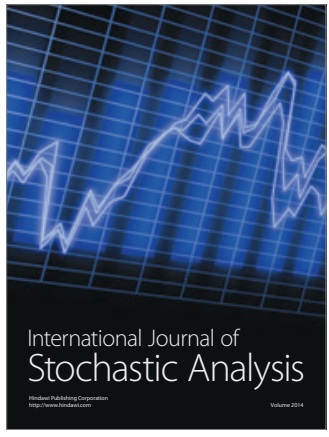

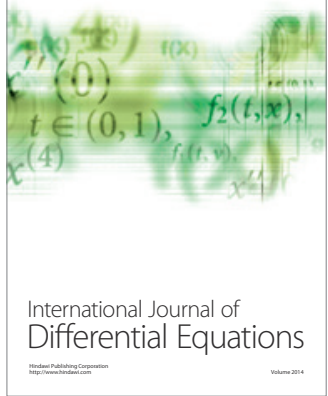
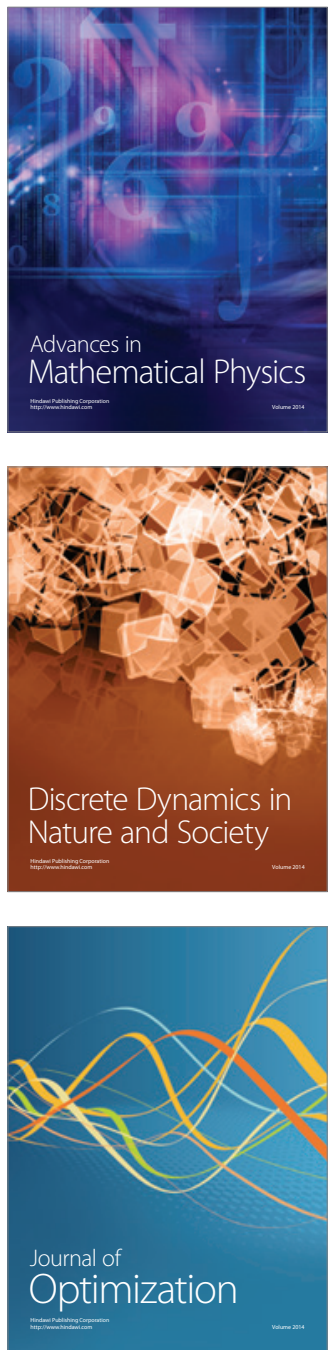